\title{
SOLUTIONS TO NONLINEAR HYPERBOLIC CAUCHY PROBLEMS WITHOUT CONVEXITY CONDITIONS $\left.{ }^{(}\right)$
}

\author{
BY \\ DONALD P. BALLOU
}

\begin{abstract}
This paper is concerned with the existence of weak solutions to certain nonlinear hyperbolic Cauchy problems. A condition on the curves of discontinuity is used which guarantees uniqueness in the class of piecewise smooth weak solutions. The method of proof is geometric in nature and is constructive in the manner of A. Douglis and Wu Cho-Chün; that is, for certain types of initial data the method of characteristics is employed to construct piecewise smooth weak solutions. A limiting process is then used to obtain existence for bounded, measurable initial data. The solutions in some cases exhibit interesting, new phenomena. For example, a certain class of initial data having one jump gives rise to a solution having a curving contact discontinuity which does not enter the region of intersecting characteristics.
\end{abstract}

1. Introduction. In this paper we consider existence of global weak solutions to the Cauchy problem

$$
\begin{gathered}
\partial F(u) / \partial t+\partial G(u) / \partial x=0, \\
u(0, x)=u^{0}(x),
\end{gathered}
$$

where $u^{0}(x)$ is bounded and measurable on $R^{1}$. The basic assumptions on $F$ and $G$ are: I. $F, G \in C^{2}\left(R^{1}\right)$; II. $F^{\prime}>0$ on $R^{1}$; III. Let $H(u)=G^{\prime}(u) / F^{\prime}(u)$. Then $H^{\prime}(u)$ vanishes at a finite number of points and changes sign at these points.

The type of weak solution we shall work with is given by the following definition.

Definition 1.1. A bounded measurable function $u=u(t, x)$ defined on $R_{+}^{2}=$ $\{(t, x):-\infty<x<\infty, t \geqq 0\}$ is a weak solution to the Cauchy problem (1.1), (1.2) provided

$$
\int_{0}^{\infty} \int_{-\infty}^{\infty}\left\{F[u(t, x)] \frac{\partial \phi}{\partial t}+G[u(t, x)] \frac{\partial \phi}{\partial x}\right\} d x d t+\int_{-\infty}^{\infty} F\left[u^{0}(x)\right] \phi(0, x) d x=0
$$

for all $\phi=\phi(t, x) \in C_{0}^{1}\left(R_{+}^{2}\right)$.

The main result of this paper is

THEOREM (EXISTENCE). Under the hypotheses I, II, and III the Cauchy problem (1.1), (1.2) has a weak solution whenever $u^{0}(x)$ is bounded and measurable.

Received by the editors August 15, 1969 and, in revised form, February 10, 1970.

AMS 1970 subject classifications. Primaty 35D05, 35F25, 35L60, 35L65; Secondary 35A 30.

Key words and phrases. Hyperbolic partial differential equations, nonlinear Cauchy problems, global weak solutions, shocks, contact discontinuities, rarefaction waves, method of characteristics, convexity conditions.

(1) This work was done under grant GP-5758 of the National Science Foundation administered by the Office of Research Administration of the University of Michigan.

Copyright (C) 1970, American Mathematical Society 
This theorem is an extension of several previous results. In the case $F(u) \equiv u$ Oleǐnik [4] and Douglis [2] obtained existence by requiring $G^{\prime \prime}(u)>0$. Wu [5] weakened this restriction on $G^{\prime \prime}$ and obtained existence for monotone $u^{0}(x)$. Conway and Smoller [1] obtained a result which when specialized to one space variable gives existence for sufficiently smooth $G$ provided $u^{0}(x)$ has locally bounded variation.

In order to obtain uniqueness it is necessary to impose some additional restriction on the weak solutions. One such restriction is Condition $\mathrm{E}$.

Condition E. Let $x(t)$ be any curve of discontinuity of the weak solution $u(t, x)$, and let $v$ be any number lying between $u(t, x(t)-0)$ and $u(t, x(t)+0)$. Then except possibly for a finite number of $t, S\left[v ; u^{-}\right] \geqq S\left[u^{+} ; u^{-}\right]$, where $u^{ \pm}=$ $u(t, x(t) \pm 0)$ and

$$
S[w ; z]=(G(w)-G(z)) /(F(w)-F(z)), \quad w \neq z .
$$

Some insight into the implications of Condition $\mathrm{E}$ is given by the following theorem.

THEOREM 1.1. Let $x=x(t) \in C^{\prime}([0, T])$ be a curve of discontinuity of $u(t, x)$ which satisfies Condition $\mathrm{E}$ and the Rankine-Hugoniot condition $\left({ }^{2}\right)$. Then

$$
H\left(u^{-}\right) \geqq \frac{d x(t)}{d t} \geqq H\left(u^{+}\right) .
$$

Proof. Condition E implies that $S\left[v ; u^{-}\right] \geqq S\left[u^{+} ; u^{-}\right] \geqq S\left[v ; u^{+}\right]$. Inequality (1.4) is obtained from the above by taking $\lim _{v \rtimes u^{-}} S\left[v ; u^{-}\right]$and $\lim _{v>u^{+}} S\left[v ; u^{+}\right]$, where for the sake of argument assume that $u^{-}<u^{+}$.

REMARKS. This result shows that the curves of discontinuity which Condition $\mathrm{E}$ allows are either shocks in the sense of Lax [3] or else are contact discontinuities. To see this we note that $H\left[u^{0}(x)\right]=G^{\prime}\left[u^{0}(x)\right] / F^{\prime}\left[u^{0}(x)\right]$ is the slope of the characteristic passing through $(0, x)$ carrying the value $u^{0}(x)$. Later we shall show that for relatively simple $u^{0}(x)$ allowing $H^{\prime}$ to vanish results in a piecewise smooth weak solution satisfying Condition $\mathrm{E}$ which has a curve of discontinuity that never enters the region of intersecting characteristics. This solution involves a fanlike function, not previously exhibited, which has a boundary with nonconstant slope. Since this boundary is a contact discontinuity, in order to find a weak solution for general $G(u)$ and $u^{0}(x)$, it is necessary to weaken the Lax shock condition.

The importance of Condition $\mathrm{E}$ is shown by the following theorem, the proof of which is a modification of work done by $\mathrm{Wu}$ [5].

TheOREM (UNIQUeNess). Let $u=u(t, x)$ and $v=v(t, x)$ be piecewise smooth functions which are weak solutions to (1.1), (1.2) and which satisfy Condition $\mathrm{E}$. Then $u(t, x) \equiv v(t, x)$.

$\left.{ }^{2}\right)$ The Rankine-Hugoniot condition holds along $x(t)$ provided $d x(t) / d t=S\left[u^{+} ; u^{-}\right]$. 
Definition 1.2. By a solution to (1.1), (1.2) we mean any piecewise smooth function defined on $R_{+}^{2}$ which is a weak solution and which satisfies Condition $\mathrm{E}$.

REMARKS. By a piecewise smooth function we mean a bounded function $u(t, x)$ that is $C^{\prime}$ on $R_{+}^{2}$ except along a finite number of curves which are themselves $C^{\prime}$. These curves can intersect at their endpoints, and except possibly at these endpoints the right and left limits of $u(t, x)$ exist and are continuous. In this paper we normalize the piecewise smooth functions by making them continuous from the left, i.e. $u(t, x)=u(t, x-0)$.

Also, frequent use will be made of the following well-known result (cf. [4]).

THEOREM 1.2. Let $u(t, x)$ be piecewise smooth and satisfy $u(0, x)=u^{0}(x)$. Then $u(t, x)$ is a weak solution to (1.1), (1.2) if, and only if,

(i) equation (1.1) holds in the usual sense at points of smoothness;

(ii) the Rankine-Hugoniot condition holds along curves of discontinuity.

2. Technical results when $H^{\prime}$ has one zero. In this section we establish several existence results when hypothesis III is replaced by hypothesis III': there exists a number $a$ such that $H^{\prime}(u)<0, u<a ; H^{\prime}(a)=0 ; H^{\prime}(u)>0, u>a$. This case not only illustrates the nature of the difficulties involved in the general one but also is needed in order to establish the general existence theorem. Furthermore, the solutions exhibit interesting behavior which is found only when $H^{\prime}$ is allowed to vanish. In particular, curving contact discontinuities which do not enter the region of intersecting characteristics arise under hypothesis III'.

The first part of this section is devoted to several results of a technical nature.

Definition 2.1. Let $\eta<a$ be given and define $\eta^{*}=\eta^{*}(\eta)$ by

$$
\eta^{*}=\sup \{u>\eta: S(v ; \eta)>S(u ; \eta) \forall v \in(\eta, u)\}
$$

Let $\eta>a$ be given and define $\eta_{*}=\eta_{*}(\eta)$ by

$$
\eta_{*}=\inf \{u<\eta: S(v ; \eta)>S(u ; \eta) \forall v \in(u, \eta)\} \text {. }
$$

We remark that the above sets are nonempty (they both contain $a$ ) and that $\eta^{*}=+\infty$ and $\eta_{*}=-\infty$ are possible. The next several lemmas establish certain properties of $\eta^{*}$. Analogous statements can be made for $\eta_{*}$, the proofs being similar to those for $\eta^{*}$.

Lemma 2.1. For each $u \in\left(\eta, \eta^{*}\right)$

$$
H(\eta)>S(u ; \eta)>H(u) .
$$

Proof. Fix $u_{0} \in\left(\eta, \eta^{*}\right)$. From Definition 2.1 it follows that

$$
S(v ; \eta)>S\left(u_{0} ; \eta\right)>S\left(v ; u_{0}\right), \quad v \in\left(\eta, u_{0}\right) .
$$

Take the limit as $v \searrow \eta$ of the left-hand term and the limit as $v \nearrow u_{0}$ of the righthand term to get $H(\eta) \geqq S\left(u_{0} ; \eta\right) \geqq H\left(u_{0}\right)$. By the mean value theorem $S\left(u_{0} ; \eta\right)$ 
$=H(\xi)$, where, as is easily seen, $\eta<\xi<a$. Since $H^{\prime}<0$ on $(-\infty, a), H(\eta)>H(\xi)$ $=S\left(u_{0}, \eta\right)$. The second inequality is handled similarly.

Corollary 1. $(d S / d u)(u ; \eta)<0, u \in\left(\eta, \eta^{*}\right)$.

Proof. $d S / d u=\left(F^{\prime}(u) /(F(u)-F(\eta))\right)[H(u)-S(u ; \eta)]<0$.

Corollary 2. $H\left(\eta^{*}\right)<H(\eta)$.

Lemma 2.2. Let $\eta<a$ be given, and suppose that $\eta^{*}<\infty$. Then $S\left(\eta^{*} ; \eta\right)=H\left(\eta^{*}\right)$.

Proof. From (2.1) and the continuity in $v$ of $S(v ; \eta)$ and $H(v), S\left(\eta^{*} ; \eta\right) \geqq H\left(\eta^{*}\right)$. If equality did not hold, then there would be an $\bar{\eta}>\eta^{*}$ such that $d S(v ; \eta) / d v<0$, $v \in(\eta, \bar{\eta})$, and hence $S(v ; \eta)>S(\bar{\eta} ; \eta) \forall v \in(\eta, \bar{\eta})$, which implies that $\bar{\eta} \leqq \eta^{*}$, a contradiction.

LeMma 2.3. Let $\eta_{1}, \eta_{2}$ be given, $\eta_{1}<\eta_{2}<a$. If $\eta_{2}^{*}<\infty$, then $\eta_{2}^{*}<\eta_{1}^{*}$.

Proof. If $\eta_{1}^{*}=\infty$, the lemma is obviously true. If $\eta_{1}^{*}<\infty$, suppose that $\eta_{1}^{*} \leqq \eta_{2}^{*}$. By Lemma $2.1 S\left(\eta_{1}^{*} ; \eta_{2}\right) \geqq H\left(\eta_{1}^{*}\right)$ while by Lemma $2.2 H\left(\eta_{1}^{*}\right)=S\left(\eta_{1}^{*} ; \eta_{1}\right)$. Since $\eta_{1}<\eta_{2}<\eta_{1}^{*}$, from Condition $\mathrm{E}$ it follows that $S\left(\eta_{1}^{*} ; \eta_{1}\right)>S\left(\eta_{2} ; \eta_{1}^{*}\right)$. Hence $S\left(\eta_{1}^{*} ; \eta_{2}\right)>S\left(\eta_{2} ; \eta_{1}^{*}\right)=S\left(\eta_{1}^{*} ; \eta_{2}\right)$, obviously a contradiction.

LEMMA 2.4. Let $\eta<a$ be given, and suppose that $\eta^{*}<\infty$. Then $\eta^{*}$ is the only zero of $\phi(u)=S(u ; \eta)-H(u), u>\eta$.

Proof. By Lemma $2.2 \phi\left(\eta^{*}\right)=0$, and by Lemma $2.1 \phi(u)>0$ on $\left(\eta, \eta^{*}\right)$. It is easily seen that $\phi^{\prime}\left(\eta^{*}\right)=-H^{\prime}\left(\eta^{*}\right)<0$. If the lemma were false, then there is a $\xi>\eta^{*}$ such that $\phi(\xi)=0$. But $\phi^{\prime}\left(\eta^{*}\right)<0$ implies $\eta^{*}$ is an isolated zero, and hence $\xi$ can be chosen so that $\phi(u)<0$ on $\left(\eta^{*}, \xi\right)$. Therefore $\phi^{\prime}(\xi) \geqq 0$, whereas from the expression for $\phi^{\prime}(u)$ it is seen that $\phi^{\prime}(\xi)<0$, a contradiction.

LEMMA 2.5. Let $\zeta$ denote the extended, real-valued function with domain $(-\infty, a)$ given by $\zeta(\eta)=\eta^{*}$. Then $\zeta$ is $C^{\prime}$ at those points $\eta$ for which $\eta^{*}<\infty$.

Proof. Let $\eta_{0}<a$ be given, $\eta_{0}^{*}<\infty$. Form the function $\Phi(u, v)=S(v ; u)-H(v)$, where $(u, v) \in(-\infty, a) \times(a, \infty)$. Note that $\partial \Phi / \partial u$ and $\partial \Phi / \partial v$ exist and are continuous, and furthermore $\partial \Phi / \partial v$ does not vanish in some neighborhood of $\left(\eta_{0}, \eta_{0}^{*}\right)$, a consequence of Lemma 2.2. Hence by the implicit function theorem there is an open interval $I, \eta_{0} \in I$, and a function $v=\alpha(u) \in C^{\prime}(I)$ such that $\Phi(u, \alpha(u))=0, u \in I$. From Lemma 2.4 it follows that $\alpha(u) \equiv \zeta(u)$ on $I$.

At this stage we can state and prove a theorem which guarantees existence of the solution if $u^{0}(x)$ is a step function with one jump. It should be noted that Theorem 2.1 allows us to construct the solution locally for an arbitrary step function. The several theorems that follow it permit us to continue the construction. In the following let $h_{1}$ be the inverse of $H$ restricted to $(-\infty, a)$, and let $h_{2}$ be the inverse of $H$ restricted to $(a, \infty)$. 
THEOREM 2.1. The Cauchy problem

$$
\begin{array}{rlrl}
\partial F(u) / \partial t & +\partial G(u) / \partial x & =0, \\
u(0, x)=\alpha, & x \leqq 0, \\
=\beta, & x>0,
\end{array}
$$

where $\alpha$ and $\beta$ are constants, $\alpha, \beta \neq a$, has a solution, $\left({ }^{3}\right)$ where the explicit form is given below for all possible values of $\alpha$ and $\beta$.

Case 1. $a<\alpha<\beta$,

$$
\begin{aligned}
u(t, x) & =\alpha, & & x \leqq H(\alpha) t, \\
& =h_{2}(x / t), & & H(\alpha) t<x \leqq H(\beta) t, \\
& =\beta, & & H(\beta) t<x .
\end{aligned}
$$

Case 2. $\alpha<\beta \leqq \alpha^{*}(\alpha<a)$,

$$
\begin{aligned}
u(t, x) & =\alpha, & & x \leqq S(\beta ; \alpha) t, \\
& =\beta, & & x>S(\beta ; \alpha) t .
\end{aligned}
$$

Case 3. $\alpha<\alpha^{*}<\beta(\alpha<a)$,

$$
\begin{aligned}
u(t, x) & =\alpha, & & x \leqq H\left(\alpha^{*}\right) t, \\
& =h_{2}(x / t), & & H\left(\alpha^{*}\right) t<x \leqq H(\beta) t, \\
& =\beta, & & H(\beta) t<x .
\end{aligned}
$$

Case 4. $\beta<\alpha<a$,

$$
\begin{aligned}
u(t, x) & =\alpha, & & x \leqq H(\alpha) t, \\
& =h_{1}(x / t), & & H(\alpha) t<x \leqq H(\beta) t, \\
& =\beta, & & H(\beta) t<x .
\end{aligned}
$$

Case 5. $\alpha_{*} \leqq \beta<\alpha$,

$$
\begin{aligned}
u(t, x) & =\alpha, & & x \leqq S(\alpha ; \beta) t, \\
& =\beta, & & x>S(\alpha ; \beta) t .
\end{aligned}
$$

Case 6. $\beta<\alpha_{*}<\alpha(a<\alpha)$,

$$
\begin{aligned}
u(t, x) & =\alpha, & & x \leqq H\left(\alpha_{*}\right) t, \\
& =h_{1}(x / t), & & H\left(\alpha_{*}\right) t<x \leqq H(\beta) t, \\
& =\beta, & & H(\beta) t<x .
\end{aligned}
$$

Proof. The proof consists of applying Theorem 1.2 to each case separately. Use is made of the above lemmas to show that the functions are weak solutions and also that Condition E holds. The details are straightforward and are omitted.

(3) See Definition 1.2 for our convention concerning the use of the word solution. 
Lemma 2.6. Let $x=x(t) \in C^{2}([0, \infty))$ satisfy $x(0)=0$, and suppose that $d^{2} x / d t^{2}<0$ for all $t \in[0, \infty)$. For each $\bar{t} \geqq 0$ let $L(\bar{t})$ be the half line

and let

$$
L(\bar{t}): x=x^{\prime}(\bar{t})(t-\bar{t})+x(\bar{t}), \quad t \geqq \bar{t},
$$

Put

$$
\Lambda=\Lambda(x(t))=\{L(\bar{t}): 0<\bar{t}<\infty\}
$$

$$
D=\left\{(t, x): t>0 \text { and } x(t)<x \leqq x^{\prime}(0) t\right\} .
$$

Then every point $(t, x) \in D$ lies on exactly one member of $\Lambda$. Furthermore, if $f=f(t) \in C^{\prime}([0, \infty))$, then the function $u=u(t, x)$ defined on $D$ by $u(t, x)=f\left(t_{1}\right)$, where $(t, x)$ lies on $L\left(t_{1}\right)$, satisfies $u \in C^{\prime}(D)$.

Proof. Fix $\left(t_{0}, x_{0}\right) \in D$, and form the function

$$
\phi(t)=\left(x(t)-x_{0}\right) /\left(t-t_{0}\right)-x^{\prime}(t) .
$$

Since $\phi(0)<0$ and $\phi(t) \rightarrow+\infty$ as $t \nearrow t_{0}$, there is some $t_{1} \in\left(0, t_{0}\right)$ such that $\phi\left(t_{1}\right)=0$, which proves the first part of the theorem. To see that $t_{1}$ is unique, note that $\phi^{\prime}>0$ at any zero of $\phi$. Since $\phi$ is $C^{1}$, it follows that $t_{1}$ must be unique.

To see that $u(t, x) \in C^{\prime}$, explicitly construct the derivatives using the definition of $u$ and note that the resulting expressions are continuous.

LEMMA 2.7. $d \eta^{*} / d u<0$, where $\eta^{*}(u)=u^{*}$.

Proof. See Lemmas 2.3 and 2.5.

In order to state the next lemma and the following theorem, we need to introduce several functions and domains. Let $u_{1}=u_{1}(x) \in C^{\prime}((-\infty, 0])$ satisfy $u_{1}<a$ and $d u_{1} / d x<0$ on $(-\infty, 0)$. Let $u_{2}=u_{2}(x) \in C^{\prime}((0, \infty))$ satisfy $u_{2}>a$ and $d u_{2} / d x \geqq 0$. Put

and let

$$
\alpha_{1}=\lim _{x>0} u_{1}(x), \quad \alpha_{2}=\lim _{x \succ 0} u_{2}(x),
$$

$$
D_{1}=\left\{(t, x): t \geqq 0 \text { and } x \leqq H\left(\alpha_{1}\right) t\right\}, \quad D_{2}=\left\{(t, x): t \geqq 0 \text { and } x \geqq H\left(\alpha_{2}\right) t\right\} .
$$

On $D_{1}$ use the initial function $u_{1}(x)$ and the method of characteristics to construct the function $v_{1}=v_{1}(t, x)$. It is well known (cf. [2]) that $v_{1}(t, x) \in C^{\prime}\left(D_{1}\right)$ and that $v_{1}(t, x)$ satisfies (1.1) in the usual sense on $D_{1}$. Similarly let $v_{2}=v_{2}(t, x)$ be defined on $D_{2}$ using $u_{2}(x)$. Lastly let $v_{1}^{*}(t, x)$ be the function given by $v_{1}^{*}(t, x)=\left(\eta^{*} \circ v_{1}\right)(t, x)$.

LEMMA 2.8. The initial value problem

(2.3) $d x / d t=S\left[v_{1}^{*}(t, x) ; v_{1}(t, x)\right]$,

(2.4) $x(0)=0$

has a solution $x=x(t) \in C^{2}$ defined for all $t>0$. Furthermore $d^{2} x / d t^{2}<0$.

Proof. By Lemma 2.2 the above initial value problem is equivalent to

(2.5) $d x / d t=H\left[v_{1}^{*}(t, x)\right]$,

(2.6) $x(0)=0$. 
To see that (2.5), (2.6) has a solution locally, it suffices to notice that $H\left[v_{1}^{*}(0,0)\right]$ $=H\left(\alpha_{1}^{*}\right)<H\left(\alpha_{1}\right)$ (the last number is the slope of the boundary of $D_{1}$ ) and that $v_{1}^{*}(t, x)$ is defined on $D_{1}$. To show that the local solution $x=x(t)$ can be defined for all $t$, it is enough to prove that

(2.7) $d x(t) / d t<H\left(\alpha_{1}^{*}\right)$

for those $t>0$ for which $x(t)$ is defined. First note that $H\left(v^{*}\right)<H\left(\alpha^{*}\right)$ whenever $\alpha<v<a$, a consequence of Lemma 2.3. Then for $t>0$ sufficiently small, (2.7) holds. This is because $v_{1}(t, x(\tilde{t}))>\alpha_{1}$, a consequence of $d u_{1} / d t<0$, and hence $v_{1}^{*}(t, x(\tilde{t}))$ $<\alpha_{1}^{*}$. Also (2.7) holds for all $t>0$. For if $\bar{t}$ is the inf of those $t$ for which (2.7) does not hold, then $\bar{t}>0$, and hence $v_{1}(\bar{t}, x(\bar{t}))>\alpha_{1}$, which gives

$$
d x(\bar{t}) / d t=H\left[v_{1}^{*}(\bar{t}, x(\bar{t}))\right]<H\left(\alpha_{1}^{*}\right),
$$

a contradiction of the definition of $\bar{t}$.

To see that $x(t) \in C^{2}$, note that $x(t)$ satisfies

$$
d x(t) / d t=H\left[v_{1}^{*}(t, x(t))\right],
$$

and that by Lemma $2.5 v_{1}^{*}(t, x) \in C^{\prime}\left(D_{1}\right)$. It remains to show that $d^{2} x / d t^{2}<0$. From (2.8) it suffices to show that

$$
\frac{d v_{1}^{*}(t, x(t))}{d t}=\frac{d \eta^{*}\left[v_{1}(t, x(t))\right]}{d u} \cdot \frac{d v_{1}(t, x(t))}{d t}<0
$$

and by Lemma 2.7 this is the case provided $d v_{1} / d t(t, x(t))>0$, a result which is easily established.

We now let $\check{x}(t)$ denote the curve whose existence is guaranteed by Lemma 2.8 . In Lemma 2.6 let $x(t)$ be $\check{x}(t)$ and let $f(t)$ be $v_{1}^{*}(t, \check{x}(t))$. Denote by $\check{u}(t, x)$ the function defined on

$$
D_{3}=\left\{(t, x): t>0 \text { and } \check{x}(t)<x \leqq H\left(\alpha_{1}^{*}\right) t\right\}
$$

which is constructed in Lemma 2.6.

The following theorem, the main theorem of this section and one of the most important of this paper, shows that the desired solution to the Cauchy problem given below has a contact discontinuity, namely $\check{x}(t)$, with nonconstant slope which does not enter the region of intersecting characteristics. The solution involves the wave-like function $\breve{u}(t, x)$ constructed above. Figure 1 is a sketch of the characteristics (indicated by broken lines) of the solution $u(t, x)$ given below.

THEOREM 2.2. Let $u_{1}(x) \in C^{\prime}((-\infty, 0])$ satisfy $u_{1}<a$ and $d u_{1} / d x<0$, and let $u_{2}(x) \in C^{\prime}((0, \infty))$ satisfy $u_{2}>a$ and $d u_{2} / d x \geqq 0$. Let $\alpha_{2}=\alpha_{1}^{*}$, where $\alpha_{i}=\lim _{\Delta \rightarrow 0} \mp u_{i}(\Delta)$. Then the function

$$
\begin{aligned}
u(t, x) & =v_{1}(t, x), & & (t, x) \in \mathrm{Cl}\left[D_{1}-D_{2} \cup D_{3}\right], \\
& =\check{u}(t, x), & & (t, x) \in D_{3}, \\
& =v_{2}(t, x), & & (t, x) \in D_{2},
\end{aligned}
$$




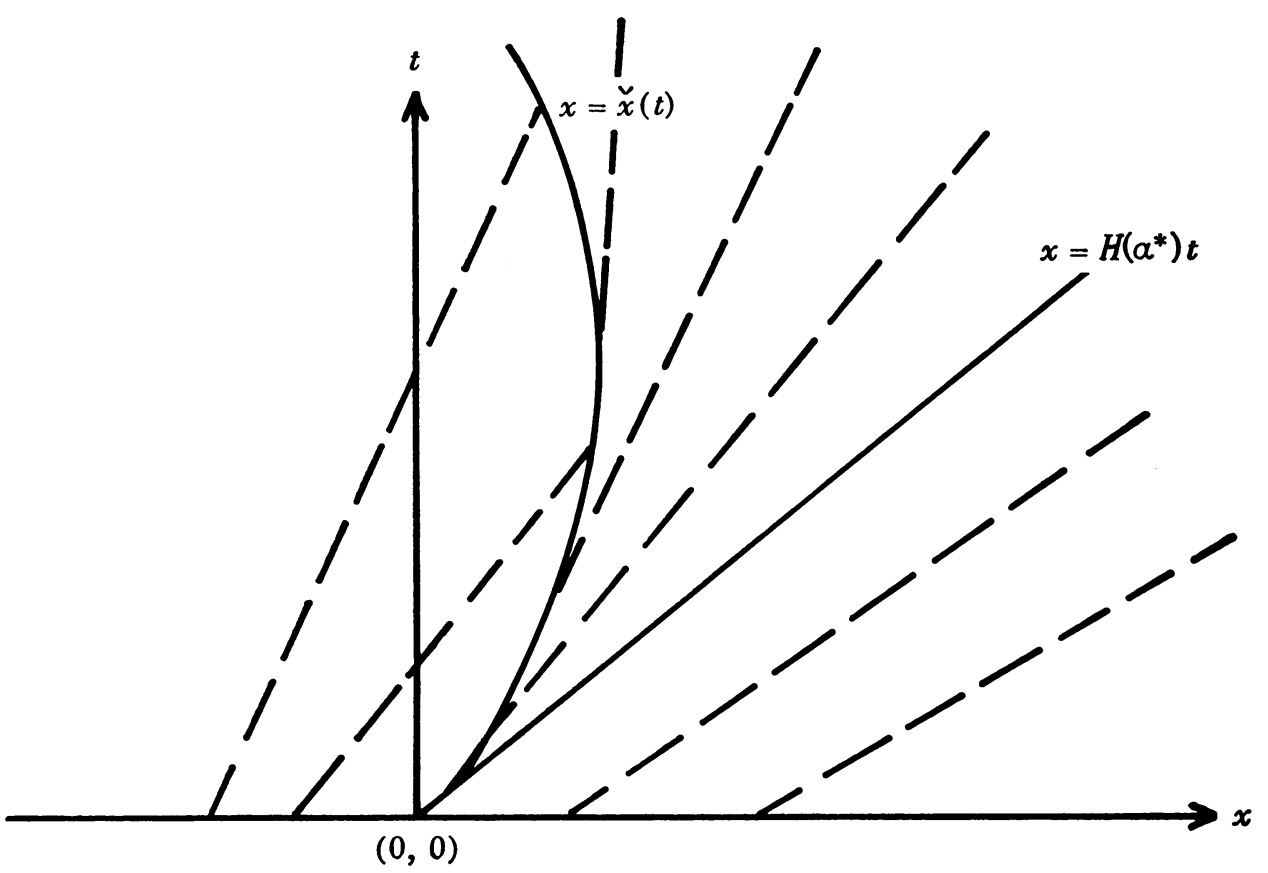

FIGURE 1

is a piecewise smooth weak solution to the Cauchy problem

$$
\begin{aligned}
\partial F(u) / \partial t & +\partial G(u) / \partial x=0 \\
u(0, x)=u_{1}(x), & x \leqq 0 \\
=u_{2}(x), & x>0,
\end{aligned}
$$

which satisfies Condition E.

Proof. Since $\check{u}(t, x) \in C^{\prime}\left(D_{3}\right), u(t, x)$ is piecewise smooth. The functions $v_{1}(t, x)$ and $v_{2}(t, x)$ satisfy $(2.11)$ in the usual sense on $D_{1}-\left(D_{2} \cup D_{3}\right)$ and $D_{2}$ respectively. To see that this is also true on $D_{3}$, fix $\left(t_{0}, x_{0}\right) \in D_{3}$ and differentiate $\check{u}(t, x)$ in the direction of $L\left(t_{1}\right)$, where $L\left(t_{1}\right)$ passes through $\left(t_{0}, x_{0}\right)$, to get the desired result.

Since $u(t, x)$ is continuous along $x=H\left(\alpha_{1}^{*}\right) t, \check{x}(t)$ is the only curve of discontinuity. From the definitions for $\check{x}(t)$ and $u(t, x)$ it is clear that the RankineHugoniot relation holds along $\check{x}(t)$, i.e.

$$
\frac{d \check{x}(t)}{d t}=\frac{G[u(t, \check{x}(t)+0)]-G[u(t, \check{x}(t)-0)]}{F[u(t, \check{x}(t)+0)]-F[u(t, \check{x}(t)-0)]}
$$

Hence by Theorem $1.2 u(t, x)$ is a weak solution of (2.11), (2.12). To see that Condition $\mathrm{E}$ holds along $\check{x}(t)$, let $v$ satisfy $v_{1}(t, \breve{x}(t))<v<v_{1}^{*}(t, \breve{x}(t))$, where $t$ is fixed but arbitrary. Then by Corollary 1 of Lemma 2.1

$$
S\left[v ; v_{1}(t, x(t))\right] \geqq S\left[v_{1}^{*}(t, x(t)) ; v_{1}(t, x(t))\right],
$$

which is Condition E. 
RemarK. Suppose $u_{1}(x)$ satisfies $u_{1}(x)>a, d u_{1} / d x>0$ while $u_{2}(x)$ satisfies $u_{2}(x)<a, d u_{2} / d x \leqq 0$. If we replace (2.3) in Lemma 2.8 with

$$
d x / d t=S\left[\left(v_{1}\right)_{*}(t, x) ; v_{1}(t, x)\right],
$$

where $\left(v_{1}\right)_{*}(t, x)=\left((\eta)_{*} \circ v_{1}\right)(t, x)$, then by a straightforward modification of the proof of that lemma, we can show that the results still hold. If $\hat{x}=\hat{x}(t)$ denotes the solution of (2.13) determined by $\hat{x}(0)=0$, let $\widetilde{D}_{3}$ and $\hat{u}(t, x)$ be defined analogously to $D_{3}$ and $\check{u}(t, x)$. Then it can easily be shown that Theorem 2.2 is valid under the above assumptions on $u_{1}$ and $u_{2}$ provided $\alpha_{1}^{*}, D_{3}, \check{u}$ are replaced by $\left(\alpha_{1}\right)_{*}, \tilde{D}_{3}, \hat{u}$. Similar comments hold for the next two theorems. These theorems are variants of Theorem 2.2.

ThEOREM 2.3. Let $u_{1}(x)$ and $u_{2}(x)$ be as in Theorem 2.2, and let $\alpha_{2}<\alpha_{1}^{*}$. Then the Cauchy problem (2.11), (2.12) has a solution.

Proof. Let $x(t)$ be the solution, which exists locally, of the initial value problem

$$
\begin{gathered}
d x / d t=S\left[v_{2}(t, x) ; v_{1}(t, x)\right], \\
x(0)=0 .
\end{gathered}
$$

Continue $x(t)$ until any one of the following occurs:

$$
\begin{aligned}
& \text { (a) } S\left[v_{2}(t, x) ; v_{1}(t, x)\right]=H\left[v_{1}^{*}(t, x)\right] \text {; } \\
& \text { (b) } x=x(t) \text { meets } x=H\left(\alpha_{1}\right) t ; \\
& \text { (c) } x=x(t) \text { meets } x=H\left(\alpha_{2}\right) t ; \\
& \text { (d) } t=\infty
\end{aligned}
$$

From Corollary 1 of Lemma 2.1 it follows that initially

$$
S\left[v_{2}(t, x(t)) ; v_{1}(t, x(t))\right]>H\left[v_{1}^{*}(t, x(t))\right] .
$$

If alternative ( $2.16 \mathrm{~d})$ holds, then the desired solution is given by

$$
\begin{aligned}
u(t, x) & =v_{1}(t, x), & & x \leqq x(t), \\
& =v_{2}(t, x), & & x>x(t) .
\end{aligned}
$$

The fact that Condition E holds along $x(t)$ is a consequence of the fact that (2.16a) did not hold for any $t$ and hence (2.17) is valid for all $t$.

Now suppose that (2.16a) holds for some $t>0$, that $\tau$ is the least such value of $t$, and that $(2.16 \mathrm{~b})$ and $(2.16 \mathrm{c})$ do not hold for $t \in(0, \tau)$. In the strip $0 \leqq t \leqq \tau$ define the desired solution $u(t, x)$ as in (2.18). It is easily seen that the "initial" data $u_{\tau}^{0}(x)=u(\tau, x)$ satisfies the hypotheses of Theorem 2.2 , and hence that result can be used to continue the construction for all $t$.

To see that $(2.16 \mathrm{~b})$ cannot occur before $(2.16 \mathrm{a})$, note that

$$
\begin{aligned}
d x(t) / d t & =S\left[v_{2}(t, x(t)) ; v_{1}(t, x(t))\right] \\
& <S\left[v_{2}(t, x(t)) ; \alpha_{1}\right] \leqq S\left[\alpha_{2} ; \alpha_{1}\right]<H\left(\alpha_{1}\right) .
\end{aligned}
$$


The first inequality is a consequence of $d \Psi / d v(v)<0$ where $\Psi(v)=S(\gamma ; v)$ with $v \in(-\infty, \xi), \xi^{*}=\gamma$, a result which is easily established. Corollary 1 of Lemma 2.1 justifies the second inequality.

Lastly suppose alternative (2.16c) holds, and let $t_{0}$ be the inf of such $t$. Since $\left(t_{0}, x\left(t_{0}\right)\right)$ lies on the characteristic $x=H\left(\alpha_{2}\right) t, v_{2}\left(t_{0}, x\left(t_{0}\right)+0\right)=\alpha_{2}$.

Since initially $x=x(t)$ lies to the right of $x=H\left(\alpha_{2}\right) t$,

$$
\begin{aligned}
H\left(\alpha_{2}\right) & \geqq d x\left(t_{0}\right) / d t=S\left[v_{2}\left(t_{0}, x\left(t_{0}\right)\right) ; v_{1}\left(t_{0}, x\left(t_{0}\right)\right)\right] \\
& \geqq H\left[v_{1}^{*}\left(t_{0}, x\left(t_{0}\right)\right)\right] .
\end{aligned}
$$

The last inequality holds because (2.16a) was not satisfied in $0<t<t_{0}$. Since

$$
\begin{aligned}
v_{1}^{*}(t, x(t)) & >v_{2}(t, x(t)) \quad \text { on } 0 \leqq t<t_{0}, \\
H\left[v_{1}^{*}\left(t_{0}, x\left(t_{0}\right)\right)\right] & \geqq H\left[v_{2}\left(t_{0}, x\left(t_{0}\right)\right)\right]=H\left(\alpha_{2}\right) .
\end{aligned}
$$

The inequalities (2.19) and (2.20) together imply that equality holds in (2.20), which yields $v_{1}^{*}\left(t_{0}, x\left(t_{0}\right)\right)=\alpha_{2}$. Hence Theorem 2.2 can be applied to complete the proof.

THEOREM 2.4. Let $u_{1}(x) \equiv \alpha_{1}$, a constant, on $(-\infty, 0)$, and let $u_{2}(x) \in C^{\prime}((0, \infty))$ satisfy $d u_{2} / d x \geqq 0$ on $(0, \infty)$. If $\alpha_{1}<a<\alpha_{2} \leqq \alpha_{1}^{*}$, then the Cauchy problem (2.12), (2.13) has a solution.

Proof. The proof is similar to that of Theorem 2.3. The details are omitted.

REMARK. At this stage it would be desirable to consider the Cauchy problem (2.11), (2.12) with restrictions of the following type on $u^{0}(x): u_{1}(x)<a, u_{2}(x)<a$, $d u_{1} / d x<0, d u_{2} / d x<0$. However cases of this type have been handled using the method of characteristics by Douglis [2], and the reader is referred to his paper for details.

3. Existence when $H^{\prime}$ has one zero. In this section we establish the existence of a piecewise smooth, global weak solution satisfying Condition E for arbitrary step data under hypothesis III', and then use this result to prove existence for bounded measurable initial data. To establish existence for step initial data, we first define the notion of "admissible initial data" and then show that solutions exist when the data is admissable. The reason for doing this is that if $u^{0}(x)$ is a step function, then by using Theorem 2.1 we can construct the desired solution $u(t, x)$ in some strip of width $t_{0}>0$. Since $u\left(t_{0}, x\right)$ is admissible, the construction can be continued.

Definition 3.1. Admissible initial data. A function $u^{0}=u^{0}(x)$ defined on $(-\infty, \infty)$ is admissible if

(i) $u^{0}(x)$ is bounded on $R^{1}$ and $u^{0}(x) \neq a$ on $R^{1}$;

(ii) $u^{0}(x) \in C^{\prime}\left(R^{1}\right)$ except at a finite number of points;

(iii) if $u^{0}(x-0)<a$, then $u^{0}(x-0) \leqq u^{0}(x+0) \leqq\left[u^{0}(x-0)\right]^{*}$; if $u^{0}(x-0)>a$, then $u^{0}(x-0) \geqq u^{0}(x+0) \geqq\left[u^{0}(x-0)\right]_{*}$;

(iv) if $u^{0}(x)$ is continuous on $I$, then $H\left[u^{0}(x)\right]$ is an increasing function of $x, x \in I$. 
Definition 3.2. A jump from $\alpha$ to $\beta$ in a function $f(x)$ is called an admissible jump if for every $v$ between $\alpha$ and $\beta, S(v ; \alpha) \geqq S(\beta ; \alpha)$.

RemarKs. Notice that each jump of an admissible function gives rise to a shock. (Fans and combinations of shocks with fans are ruled out.) Also since $H\left[u^{0}(x)\right]$ is increasing on any interval $I$ on which $u^{0}(x)$ is continuous, the characteristics determined by $u^{0}(x)$ cannot intersect.

Definition 3.3. Let $x(t)$ be a shock of the function $u(t, x)$; that is, a curve of discontinuity that satisfies Condition $\mathrm{E}$ and the Rankine-Hugoniot condition.

(i) $x(t)$ is a type I shock provided that for each $t_{0} \geqq 0, x(t)$ enters and remains within the closed wedge that has $\left(t_{0}, x\left(t_{0}\right)\right)$ as its vertex and sides with slopes $H\left[u\left(t_{0}, x\left(t_{0}\right)+0\right)\right]$ and $H\left[u\left(t_{0}, x\left(t_{0}\right)-0\right)\right]$.

(ii) $x(t)$ is a type II shock provided

(a) $d^{2} x(t) / d t^{2} \neq 0, \quad t \geqq 0$.

(b) $d x(t) / d t=H\left[\{u(t, x(t)-0)\}^{*}\right]$ or $d x(t) / d t=H\left[\{u(t, x(t)-0)\}_{*}\right]$.

(iii) $x(t)$ is a shock of mixed type provided that there exist disjoint intervals $I_{1}, I_{2}$ such that (i) holds on $I_{1}$ while (ii) holds on $I_{2}$.

Definition 3.4. A function $u=u(t, x)$ defined on a wedge-shaped region $D \subset R_{+}^{2}$ with vertex at $(0, P)$ is a type I fan provided $u(t, x)=g((x-P) / t),(t, x) \in D$, where $g$ is a strictly monotonic function of class $C^{\prime}$ on the domain of $(x-P) / t$.

Definition 3.5. Let $x(t)$ be a type II shock originating at $(0, P)$. Then the functions $\check{u}(t, x)$ and $\hat{u}(t, x)$ defined on $\left\{(t, x): t>0\right.$ and $\left.x(t)<x<H\left[u^{0}(P+0) t+P\right]\right\}$ are type II fans.

To establish existence for $u^{0}(x)$ admissible, we need the following lemmas.

LEMMA 3.1. Let $u(t, x)$ be a piecewise smooth weak solution with admissible initial data $u^{0}(x)$. Let $x_{1}(t)$ and $x_{2}(t)$ be two shocks which intersect at $t=\bar{t}$ and which satisfy Condition $\mathrm{E}$ on $0 \leqq t \leqq \bar{t}$. Then the jump in $u(\bar{t}, x)$ at $\left(\bar{t}, x_{1}(\bar{t})\right)$ is admissible.

Proof. Suppose that $x_{1}(t)<x_{2}(t)$ on $0 \leqq t \leqq \bar{t}$. Then let

$$
\begin{aligned}
& \alpha_{1}=u\left(\bar{t}, x_{1}(\bar{t})-0\right) ; \\
& \alpha_{2}=\lim _{t \nearrow t} u\left(t, x_{1}(t)+0\right)=\lim _{t \nearrow t} u\left(t, x_{2}(t)-0\right) ; \\
& \alpha_{3}=u\left(\bar{t}, x_{2}(\bar{t})+0\right) .
\end{aligned}
$$

If $\alpha_{1}<a$, it must be shown that $\alpha_{1}<\alpha_{3} \leqq \alpha_{1}^{*}$, while if $\alpha_{1}>a$, it must be shown that $\alpha_{1}>\alpha_{3} \geqq\left(\alpha_{1}\right)_{*}$. The first case is treated. Since $\alpha_{1}<a$, then $\alpha_{1}<\alpha_{2} \leqq \alpha_{1}^{*}$. If $\alpha_{2}<a$ should hold, then $\alpha_{2}<\alpha_{3} \leqq \alpha_{2}^{*}$. But $\alpha_{1}<\alpha_{2}<a$ implies $\alpha_{1}^{*}>\alpha_{2}^{*}$, which gives $\alpha_{1}<\alpha_{3}<\alpha_{1}^{*}$. If $\alpha_{2}>a$, then $\alpha_{2}>\alpha_{3} \geqq\left(\alpha_{2}\right)_{*}$. Since $\alpha_{1}^{*} \geqq \alpha_{2}, \alpha_{1}^{*}>\alpha_{3}$. It remains to show that $\alpha_{3}>\alpha_{1}$, which is true if $\left(\alpha_{2}\right)_{*}>\alpha_{1}$. But

$$
H\left(\alpha_{1}\right)>H\left(\alpha_{1}^{*}\right) \geqq H\left(\alpha_{2}\right)>H\left[\left(\alpha_{2}\right)_{*}\right] .
$$

Since $\alpha_{1},\left(\alpha_{2}\right)_{*}<a$, by the above $\alpha_{1}<\left(\alpha_{2}\right)_{*}$. 
LeMMA 3.2. Let $u(t, x)$ be a solution of the Cauchy problem with admissible initial data $u^{0}(x)$. Then for each $t_{0}>0, u\left(t_{0}, x\right)$ is admissible.

Proof. The proof is by induction on the number of discontinuities of $u^{0}(x)$. First let $u^{0}(x)$ have one discontinuity, which must give rise to a shock $x(t)$. From the results of $\S 2$ it is easily seen that $u\left(t_{0}, x\right), t_{0}>0$, has the desired monotonicity and differentiability properties to the left and right of $\left(t_{0}, x\left(t_{0}\right)\right)$. Since Condition $\mathrm{E}$ holds across $x(t), u\left(t_{0}, x\right)$ must be admissible.

Now let $u^{0}(x)$ have $n$ jumps, and let $\bar{t}>0$ be the least value of $t$ at which two shocks intersect, say at $(\bar{t}, \bar{x})$. Clearly $u\left(t_{0}, x\right)$ is admissible, $0<t_{0}<\bar{t}$. By Lemma 3.1 the jump at $(\bar{t}, \bar{x})$ must be admissible, and hence $u(\bar{t}, x)$ is an admissible function with at most $(n-1)$ jumps. Now apply the induction hypothesis to complete the proof.

THEOREM 3.1. Let $u^{0}(x)$ be an admissible function. Then the Cauchy problem under hypotheses I, II, III' has a solution.

Proof. The proof is by induction on the number of jumps in $u^{0}(x)$. Straightforward modifications of the results in $\$ 2$ handle the case of one jump. Now suppose that there are $n$ jumps, say at $P_{1}<P_{2}<\cdots<P_{n}$. Consider the Cauchy problem

$$
\begin{array}{rlrl}
\partial F(v) / \partial t & +\partial G(v) / \partial x=0, & \\
v(0, x)=v^{0}(x) & =u^{0}(x), & & x \leqq P_{n}, \\
& =u^{0}\left(P_{n}-0\right), & & x>P_{n} .
\end{array}
$$

Since $v^{0}(x)$ is an admissible function with $(n-1)$ jumps, by induction there is a function $v=v(t, x)$ that is a solution to (3.1), (3.2).

On the region

$$
D=\left\{(t, x): t \geqq 0 \text { and } x>H\left[u^{0}\left(P_{n}+0\right)\right] t+P_{n}\right\}
$$

construct the continuous piecewise smooth function $w_{1}=w_{1}(t, x)$ using the method of characteristics with $u^{0}(x)$ on $x>P_{n}$. Let $w_{2}=w_{2}(t, x)$ be defined on $R_{+}^{2}$ by

$$
w_{2}(t, x)=w_{1}(t, x), \quad \text { if }(t, x) \in D \text { and either } v(t, x)<a \text { and }
$$

$$
w_{1}(t, x) \leqq v^{*}(t, x) \text { or } v(t, x)>a \text { and } w_{1}(t, x) \geqq v_{*}(t, x) \text {, }
$$

$$
=v^{*}(t, x), \quad \text { if } v(t, x)<a \text { and either }(t, x) \notin D \text { or } w_{1}(t, x)>v_{1}^{*}(t, x) \text {, }
$$

$$
=v_{*}(t, x), \quad \text { if } v(t, x)>a \text { and either }(t, x) \notin D \text { or } w_{1}(t, x)<v_{*}(t, x) \text {. }
$$

Let $x=x(t)$ be the local solution of $d x / d t=S\left[w_{2}(t, w) ; v(t, x)\right], x(0)=P_{n}$, and extend $x(t)$ until it meets a shock of $v(t, x)$, say at $\bar{t}$, where $\bar{t}=\infty$ is possible.

First suppose that for $0<t<\bar{t}, x(t)$ is a type I shock. Then form the function $u$ given by

$$
\begin{aligned}
u(t, x) & =v(t, x), & & 0 \leqq t \leqq \bar{t}, x \leqq x(t) \\
& =w_{1}(t, x), & & 0 \leqq t \leqq \bar{t}, x>x(t)
\end{aligned}
$$


Observe that $u(t, x)$ has the desired properties on $0 \leqq t \leqq \bar{t}$. To see this it suffices to note that since $x(t)$ is a type I shock, by (3.3) $w_{2}(t, x)=w_{1}(t, x)$. If $\bar{t}<\infty$, then by Lemmas 3.1 and $3.2 u(\bar{t}, x)$ is admissible, and since $u(\bar{t}, x)$ has at most $(n-1)$ jumps, the induction hypothesis with $u(\bar{t}, x)$ as initial data along $t=\bar{t}$ can be applied to complete this part of the proof.

It remains to consider the cases when $x(t)$ is either a type II shock or else a shock of mixed type. The former case is examined, as only minor modifications are needed for the latter. Then let $\tilde{v}(t, x)$ be the type II fan determined by $v(t, x)$ and $x(t)$. Then, letting $\bar{t}$ be as before, the solution on $0 \leqq t \leqq \bar{t}$ is given by

$$
\begin{aligned}
u(t, x) & =v(t, x), & & x \leqq x(t), \quad 0 \leqq t \leqq \bar{t}, \\
& =\tilde{v}(t, x), & & x(t)<x \leqq H\left[u^{0}\left(P_{n}+0\right)\right] t+P_{n}, \quad 0 \leqq t \leqq \bar{t}, \\
& =w_{1}(t, x), & & H\left[u^{0}\left(P_{n}+0\right)\right] t+P_{n}<x, \quad 0 \leqq t \leqq \bar{t} .
\end{aligned}
$$

If $\bar{t}<\infty$, then it is easily seen that $u(\bar{t}, x)$ is an admissible function with at most $(n-1)$ jumps, and thus the induction hypothesis can be applied to complete the proof.

THEOREM 3.2. Let $u^{0}(x)$ be a step function. Then under the hypotheses, I, II, III' the Cauchy problem (1.1), (1.2) has a solution $\left({ }^{4}\right)$.

Proof. In this context a step function is a piecewise constant function which has a finite number of discontinuities. Using Theorem 2.1 it is possible to construct a solution $u(t, x)$ in some strip $0 \leqq t \leqq \tau$, where $\tau>0$ is sufficiently small so that no shocks or fans arising from the discontinuities can interact. (The boundedness of the initial data guarantees that such a $\tau$ exists.) From the explicit expressions for $u(t, x)$ given in Theorem 2.1 together with Definition 3.1 it is seen that $u\left(t_{0}, x\right)$ is admissible. Hence by Theorem 3.2 the construction can be continued to define the solution for all $t>0$.

In order to pass to the limit and obtain existence for bounded, measurable initial data, it is necessary to use an ordering principle, a statement of which is given below. The proof is a modification of work found in $\mathrm{Wu}$ [5].

Theorem 3.3 (Ordering Principle). Let $F$ and $G$ satisfy the basic hypotheses I and II only, and let $u(t, x)$ and $v(t, x)$ be piecewise smooth weak solutions satisfying Condition $\mathrm{E}$ to the Cauchy problem (1.1), (1.2), where the initial data are $u(0, x)$ and $v(0, x)$ respectively. Then $u(0, x) \leqq v(0, x) \forall x \in R^{1}$ implies $u(t, x) \leqq v(t, x) \forall(t, x) \in R_{+}^{2}$.

Once the ordering principle is available, by using Theorem 3.2 we can proceed as in Douglis [2] to establish the following existence theorem. The reader is referred to Douglis for details.

THEOREM 3.4. Let $F$ and $G$ satisfy the basic hypotheses I, II, III', and let $u^{0}(x)$ be bounded and measurable. Then the Cauchy problem (1.1), (1.2) has a global weak solution.

(4) See Definition 1.2 for our convention concerning the use of the word solution. 
4. General existence theorem. In this section we suppose that hypothesis III holds, i.e. there are points $a_{1}<a_{2}<\cdots<a_{n}$ at which $H^{\prime}$ vanishes and changes sign. For the sake of definiteness we suppose that $H^{\prime}(u)<0, u<a_{1} ; H^{\prime}(u)>0, a_{1}<u<a_{2}$; $H^{\prime}(u)<0, a_{2}<u<a_{3}$; etc. We shall first construct the desired solution when $u^{0}(x)$ is a step function with one jump and then solve (1.1), (1.2) for several special cases of $u^{0}(x)$. These will enable us to solve (1.1), (1.2) when $u^{0}(x)$ is a step function. Once this is known, the general existence theorem follows.

In this section we shall refer to the curve $\sigma$ which in the $v w$-plane is given parametrically by $v=F(u), w=G(u)$. Notice that the points $\left(F\left(a_{i}\right), G\left(a_{i}\right)\right)$ are inflection points of $\sigma$. Also let $T(u)$ denote the tangent to $\sigma$ at $\sigma(u)$.

THEOREM 4.1. The Cauchy problem (1.1), (1.2) with

$$
\begin{aligned}
u^{0}(x) & =\alpha, & x \leqq 0, \\
& =\beta, & x>0,
\end{aligned}
$$

$\alpha$ and $\beta$ constants, has a solution.

Proof. We describe how to construct the solution $u(t, x)$. To the left of the first shock let $u(t, x) \equiv \alpha$ and to the right of the last shock let $u(t, x) \equiv \beta$. When we say to fan from some number $c_{1}$ to another $c_{2}$, it should be noticed that no $a_{i}$ lies between $c_{1}$ and $c_{2}$, and hence it is possible to define the fan, since $H$ restricted to this domain has an inverse.

We shall assume that $\alpha<\beta$; the case $\beta<\alpha$ is treated similarly. Let

$$
\Sigma=\{u: \alpha<u \leqq \beta \text { and } S(v ; \alpha) \geqq S(u ; \alpha) \forall v \in(\alpha, u)\}
$$

We first suppose that $\Sigma \neq \varnothing$. Then let $\eta^{*}(\alpha ; \beta)=\sup \Sigma$. If $\eta^{*}(\alpha, \beta)=\beta$, we can shock from $\alpha$ to $\beta$ and have Condition E hold. If $\eta^{*}(\alpha ; \beta)<\beta$, we shock from $\alpha$ to $\eta^{*}(\alpha ; \beta)$ along $x=H\left[\eta^{*}(\alpha ; \beta)\right] t$. It is easily shown that $H\left[\eta^{*}(\alpha ; \beta)\right]=S\left[\eta^{*}(\alpha ; \beta) ; \alpha\right]$. To continue the construction, we notice that at the point $\sigma\left[\eta^{*}(\alpha ; \beta)\right] \sigma$ must be convex upward. Hence we can fan from $\eta^{*}(\alpha ; \beta)$ to $\alpha_{1}>\eta^{*}(\alpha ; \beta)$, where either

(i) $\alpha_{1}=\beta$; or

(ii) $\alpha_{1}$ is such that $\sigma(\beta)$ lies on $T\left(\alpha_{1}\right)$ and $S\left(v ; \alpha_{1}\right) \geqq S\left(\beta ; \alpha_{1}\right) \forall v \in\left(\alpha_{1}, \beta\right)$; or

(iii) $\alpha_{1}$ is the first number $>\eta^{*}(\alpha ; \beta)$

for which there exists $\zeta, \alpha_{1}<\zeta<\beta$ such that $T\left(\alpha_{1}\right)$ and $T(\zeta)$ coincide.

Note that if (i) holds, we are done. In case (ii) we shock from $\alpha_{1}$ to $\beta$ along $x=H\left(\alpha_{1}\right) t$ to complete the construction. For case (iii) let $\alpha_{2}=\sup \{\zeta: \zeta$ satisfies (iii) $\}$. It can be shown that $H\left(\alpha_{2}\right)=S\left(\alpha_{2} ; \alpha_{1}\right)$. Thus we can shock from $\alpha_{1}$ to $\alpha_{2}$ along $x=H\left(\alpha_{2}\right) t=H\left(\alpha_{1}\right) t$. This procedure is now repeated, but since there must be at least two $a_{i}$ 's between $\alpha_{1}$ and $\alpha_{2}$, after a finite number of such repetitions we are finished.

Lastly, if $\Sigma=\varnothing$, then $\sigma$ must be convex upward at $\sigma(\alpha)$, and hence we can fan from $\alpha$ to $\alpha_{1}$, where $\alpha_{1}$ is described as above. We then proceed as before. 
In this section it is expedient to work with a modified version of the definition of admissible function given in $\$ 3$.

Definition 4.1. A function $u^{0}=u^{0}(x)$ defined, bounded, and piecewise smooth on $R^{1}$ is said to be admissible if

(i) $u^{0}(x) \neq a_{i}, \forall x \in R^{1}, i=1,2, \ldots, n$;

(ii) if $H^{\prime}(u)>0$ on $\left(a_{i}, a_{i+1}\right)$, then for every $x$ such that $u^{0}(x) \in\left(a_{i}, a_{i+1}\right)$ and $d u^{0} / d x$ exists, $d u^{0} / d x(x) \geqq 0$;

(iii) if $H^{\prime}(u)<0$ on $\left(a_{i}, a_{i+1}\right)$, then for every $x$ such that $u^{0}(x) \in\left(a_{i}, a_{i+1}\right)$ and $d u^{0} / d x$ exists, $d u^{0} / d x(x) \leqq 0$.

In the next several theorems we show that (1.1), (1.2) has a solution when the initial data is admissible. We shall first prove this when there is one jump by using induction on the number of zeros of $H^{\prime}$. But to do this we first need to establish the desired result in several special cases. It will be noted that we do not consider those cases for which $u^{0}(x)$ is admissible and monotonic. The proofs for these cases can be found in $\mathrm{Wu}[5]$.

TheOREM 4.2. Suppose $H$ satisfies hypothesis III with $n=1$. Then the Cauchy problem (1.1), (1.2) has a solution whenever $u^{0}(x)$ is an admissible function with one jump.

Proof. Except for the removal of the restriction on the size of the jump in the initial data, the theorems in $\$ 2$ could be quoted to prove Theorem 4.2. To see how the proofs of $\S 2$ should be modified, suppose, for example, that the hypotheses of Theorem 2.2 hold except that $\beta>\alpha^{*}$. Then the function

$$
\begin{aligned}
u(t, x) & =v_{1}(t, x), & & (t, x) \in \mathrm{Cl}\left[D_{1}-\left(D_{2} \cup D_{3}\right)\right], \\
& =\check{u}(t, x), & & (t, x) \in D_{3}, \\
& =h_{2}(x / t), & & H\left(\alpha^{*}\right) t<x \leqq H(\beta) t, \\
& =v_{2}(t, x), & & x>H(\beta) t,
\end{aligned}
$$

where $h_{2}$ is the inverse of $H$ restricted to $(a, \infty)$, is the desired solution.

In the following special cases the initial data has the form

$$
\begin{aligned}
u^{0}(x) & =u_{1}^{0}(x), & & x \leqq 0, \\
& =u_{2}^{0}(x), & & x>0,
\end{aligned}
$$

where $u_{1}^{0}(x) \in C^{\prime}[(-\infty, 0)]$ and $u_{2}^{0}(x) \in C^{\prime}[(0, \infty)]$.

Let $\alpha=\lim _{x>0} u_{1}^{0}(x)$ and $\beta=\lim _{x \succ 0} u_{2}^{0}(x)$. We require that $u^{0}(x)$ be admissible, and for the sake of argument we shall assume that $\alpha<\beta$.

THEOREM 4.3. Let $H$ satisfy hypothesis III with $n=2$, and suppose that $\alpha<a_{1}<a_{2}<\beta$. If $S(v ; \alpha) \geqq S(\beta ; \alpha) \forall v \in(\alpha, \beta)$ and there exists exactly one $\gamma$ such that equality holds, then (1.1), (1.2) has a solution wherever $u^{0}(x)$ is admissible and has form (4.1). 
Proof. By Theorem 2.2 and the analogue in the case $H^{\prime}(u)>0, u<a ; H^{\prime}(a)=0$; $H^{\prime}(u)<0, u>a$, there exist solutions $u_{1}(t, x)$ and $u_{2}(t, x)$ to the Cauchy problems with

$$
\begin{aligned}
u(0, x) & =u_{1}^{0}(x), & & x \leqq 0, \\
& =\gamma, & & x>0, \\
u(0, x) & =\gamma, & & x \leqq 0, \\
& =u_{2}^{0}(x), & & x>0,
\end{aligned}
$$

respectively as the initial data. Since $u_{1}(t, H(\gamma) t)=u_{2}(t, H(\gamma) t)=\gamma$, it is easily seen that

$$
\begin{aligned}
u(t, x) & =u_{1}(t, x), & & x \leqq H(\gamma) t, \\
& =u_{2}(t, x), & & x>H(\gamma) t,
\end{aligned}
$$

has the desired properties.

RemarK. We note that $u(t, x)$ has two contact discontinuities, each originating at $(0,0)$. Below is a sketch of the shocks (given by the solid curves) and the characteristics (given by the dotted lines) of $u(t, x)$.

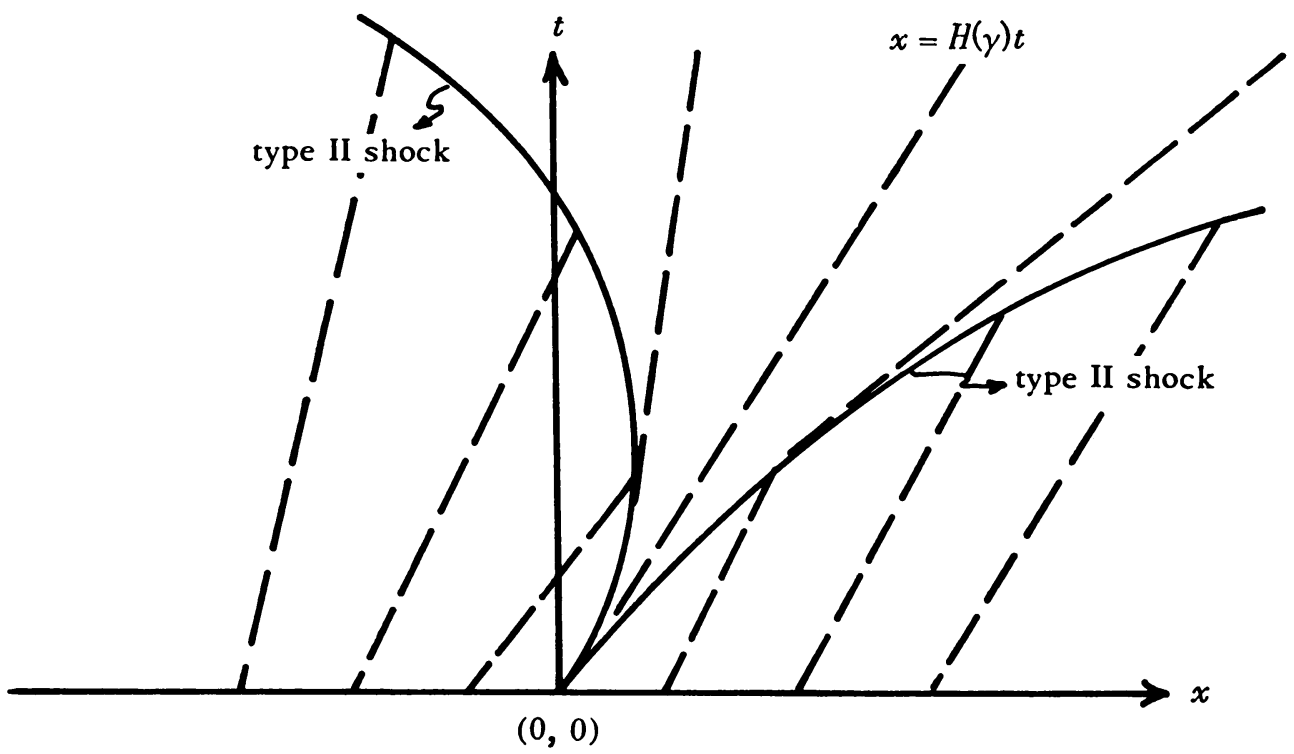

FIGURE 2

THEOREM 4.4. Let $H$ satisfy hypothesis III with $n=4$. Assume that $\alpha<a_{1}<a_{4}<\beta$, $S(v ; \alpha) \geqq S(\beta ; \alpha) \forall v \in(\alpha ; \beta)$, and that there exists $\gamma_{1} \in\left(a_{1}, a_{2}\right)$ and $\gamma_{2} \in\left(a_{3}, a_{4}\right)$ such that $S\left(\gamma_{1} ; \alpha\right)=S\left(\gamma_{2} ; \alpha\right)=S(\beta ; \alpha)$. Then (1.1), (1.2) has a solution whenever $u^{0}(x)$ is admissible and has the form (4.1). 
Proof. Let $u_{1}(t, x)$ and $u_{2}(t, x)$ be the solutions to the Cauchy problems with initial data

and

$$
\begin{aligned}
u(0, x) & =u_{1}^{0}(x), & & x \leqq 0, \\
& =\gamma_{1}, & & x>0,
\end{aligned}
$$

respectively. Let

$$
\begin{aligned}
u(0, x) & =\gamma_{2}, & & x \leqq 0, \\
& =u_{2}^{0}(x), & & x>0,
\end{aligned}
$$

$$
\begin{aligned}
u(t, x) & =u_{1}(t, x), & & x \leqq H\left(\gamma_{1}\right) t, \\
& =u_{2}(t, x), & & x>H\left(\gamma_{1}\right) t .
\end{aligned}
$$

Note that $u(t, x)$ jumps from $\gamma_{1}$ to $\gamma_{2}$ along $x=H\left(\gamma_{1}\right) t$. Since, as is easily seen, both Condition $\mathrm{E}$ and the Rankine-Hugoniot condition hold across $x=H\left(\gamma_{1}\right) t, u(t, x)$ is the desired solution.

REMARK. The shocks (indicated by solid curves) and characteristics (indicated by broken lines) for (4.2) are sketched below.

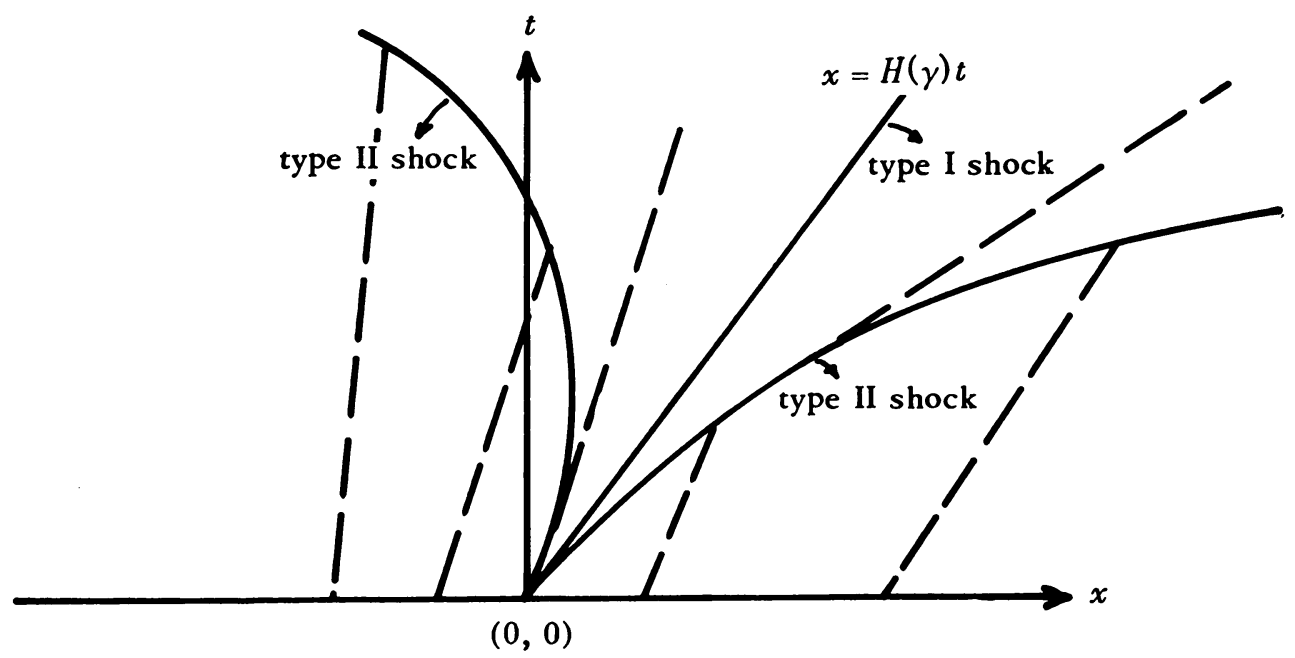

FIGURE 3

THEOREM 4.5. Let $H$ satisfy hypothesis III with $n=3$. If $\alpha<a_{1}<a_{3}<\beta$ and $S(v ; \alpha) \geqq S(\beta ; \alpha) \forall v \in(\alpha, \beta)$, then the Cauchy problem (1.1), (1.2) has a solution.

Proof. Let $x_{0}(t)$ be the solution of

$$
d x / d t=S\left[v_{2}(t, x) ; v_{1}(t, x)\right], \quad x(0)=0 .
$$

If $d x / d t(t)=H\left[v_{2}(t, x(t))\right]$ should hold for some $t$, then a type II fan is needed. Otherwise let

$$
t_{0}=\sup \left\{\tau: \text { condition } \mathrm{E} \text { holds along } x_{0}(t) \forall t \in(0, \tau)\right\} .
$$


$t_{0}=\infty$ is possible, in which case there is nothing to prove. Otherwise without loss of generality suppose that $t_{0}=0$. Hence there is a $\gamma \in\left(a_{1}, a_{2}\right)$ such that $S(\gamma ; \alpha)$ $=S(\beta ; \alpha)$.

Let $u_{1}(t, x)$ be the solution of (1.1), (1.2) with

$$
\begin{aligned}
u(0, x) & =u_{1}^{0}(x), & & x \leqq 0, \\
& =\gamma, & & x>0 .
\end{aligned}
$$

Note that $u_{1}(t, x)$ has a type II shock $x=x_{1}(t)$ lying to the left of $x=H(\gamma) t$. Next define $\tilde{u}(t, x)$ on $R_{+}^{2}$ by

$$
\begin{aligned}
\tilde{u}(t, x) & =\check{u}_{1}\left(t, x_{1}(t)+0\right), & & x \leqq x_{1}(t), \\
& =\check{u}_{1}(t, x), & & x_{1}(t)<x \leqq S(\beta ; \alpha) t, \\
& =\beta, & & S(\beta ; \alpha) t<x,
\end{aligned}
$$

where $\check{u}_{1}(t, x)$ is the type II fan lying between $x=x_{1}(t)$ and $x=S(\beta ; \alpha) t$. Let $x_{2}(t)$ be the solution of

$$
d x / d t=S\left[\tilde{u}(t, x) ; v_{2}(t, x)\right], \quad x(0)=0 .
$$

Using the defining property of $t_{0}$, it can be shown that $S(\beta ; \alpha) t>x_{2}(t)>x_{1}(t)$ for $t$ positive but sufficiently small. Continue $x_{2}(t)$ until it meets $x_{1}(t)$, say at $t=\tau$, otherwise indefinitely. The function

$$
\begin{aligned}
u(t, x) & =v_{1}(t, x), & & x \leqq x_{1}(t), \\
& =\check{u}_{1}(t, x), & & x_{1}(t)<x \leqq x_{2}(t), \\
& =v_{2}(t, x), & & x_{2}(t)<x,
\end{aligned}
$$

is the desired solution on $[0, \tau]$. Now repeat the above procedure, a process that cannot be terminated. To see this suppose otherwise. Then for some $T>0$ the solution $u(t, x)$ can be defined on $[0, T]$, but $u(T, x)$ is not admissible. However, using the fact that any shock has bounded slope, this gives a contradiction.

THEOREM 4.6. Let $H$ satisfy hypothesis III with $n$ arbitrary. Suppose that the initial data $u^{0}(x)$ is admissible, has one jump, and satisfies either $u_{1}^{0}(x)<a_{1}, u_{2}^{0}(x)>a_{n}$ or $u_{1}^{0}(x)>a_{n}, u_{2}^{0}(x)<a_{1}$. Then the Cauchy problem (1.1), (1.2) has a solution.

Proof. The case $u_{1}^{0}(x)<a_{1}, u_{2}^{0}(x)>a_{n}$ is considered. First suppose that $S(v ; \alpha)$ $\geqq S(\beta ; \alpha) \forall v \in(\alpha, \beta)$. Let $x(t)$ be the solution of

$$
d x / d t=S\left[v_{2}(t, x) ; v_{1}(t, x)\right], \quad x(0)=0 .
$$

Extend $x(t)$ until for some $\tau>0$ Condition E fails to hold for $t>\tau$. Then either

(i) $S\left[v_{2}(\tau, x(\tau)) ; v_{1}(\tau, x(\tau))\right]=H\left[v_{2}(\tau, x(\tau))\right]$ or

(ii) there exist one or more $\gamma \in\left(v_{1}(\tau, x(\tau)), v_{2}(\tau, x(\tau))\right)$ such that $S\left[\gamma ; v_{1}(\tau, x(\tau))\right]$ $=S\left[v_{2}(\tau, x(\tau)) ; v_{1}(\tau, x(\tau))\right]$.

Case (i) is handled by using a type II fan, while in case (ii) proceed as in the appropriate special case considered above. 
If $\beta$ is such that it is not possible to shock from $\alpha$ to $\beta$, then proceed as in Theorem 4.1 to find type I fans, type II fans, and contact discontinuities that when properly combined yield the desired solution.

THEOREM 4.7. Let $H$ satisfy hypothesis III with $n$ general. Let $u^{0}(x)$ be admissible and have one jump. Then the Cauchy problem (1.1), (1.2) has a solution.

Proof. The proof is by induction. The case $n=1$ is valid by Theorem 4.2. Assume that Theorem 4.7 is true if $H^{\prime}$ has $(n-1)$ zeros. Since $u^{0}(x)$ is admissible, there are integers $i, j$ such that $a_{i-1}<u_{1}^{0}(x)<a_{i}$ and $a_{j}<u_{2}^{0}(x)<a_{j+1}$, where $a_{0}=-\infty$ and $a_{n+1}=+\infty$. For the sake of argument suppose that $i \leqq j$. If either $i>1$ or $j<m$ should hold, then on $\left(a_{i-1}, a_{j+1}\right) H^{\prime}$ would have fewer than $n$ zeros, and so by induction (1.1), (1.2) has a solution. If $i=1$ and $j=n$, then the desired solution exists by Theorem 4.6 .

THEOREM 4.8. Let $H$ satisfy hypothesis III with $n$ arbitrary. If $u^{0}(x)$ is admissible, then the Cauchy problem (1.1), (1.2) has a solution.

Proof. Using Theorem 4.7 the solution can be constructed in some strip $0 \leqq t \leqq \tau, \tau>0$. Continue the solution until two shocks intersect, say at $t=t_{0}$. Since the constructed function along $t=t_{0}$ is admissible, the procedure can be repeated. It remains only to show that this process does not terminate. To establish this assume the contrary and let

$$
T=\sup \{\tau: \text { the desired solution can be defined in } 0 \leqq t \leqq \tau\} .
$$

Hence $u(T, x)$ cannot be admissible, which will lead to a contradiction. Now the number of shocks is bounded by some constant $N$ which depends only on $u^{0}(x)$; the proof of this fact is deferred. Choose an increasing sequence $\left\{t_{n}\right\}_{1}^{\infty}$ converging to $T$. Let $M>0$ be a bound for the slope of any shock. $M$ depends on $u^{0}(x)$ and $H$. At each point of the line $t=t_{n}$ where $u\left(t_{n}, x\right)$ is discontinuous, draw the two lines with slopes $M$ and $-M$ respectively. Let $\left\{I_{n_{K}}\right\}_{K=1}^{N_{n}}$ be the bounded intervals on $t=T$ cut off by these lines. Notice that $0 \leqq N_{n}<N$. Let $J_{n}=\left(\bigcup_{K=1}^{N_{n}} I_{n_{K}}\right)$. From the method

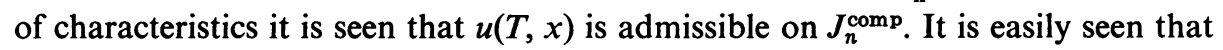
$J_{1} \supset J_{2} \supset \ldots$. Since the length of $J_{n}$ tends to zero as $n \rightarrow \infty, u(T, x)$ is admissible, a contradiction.

It remains to show that the number of shocks is bounded by some number $N$ depending only on $u^{0}(x)$. For this notice that since the characteristics carry the data $u^{0}(x)$,

$$
\underset{-\infty<x<\infty}{\operatorname{Var}} u(t, x) \leqq \operatorname{Var}_{-\infty<x<\infty} u^{0}(x), \quad 0 \leqq t<\tau .
$$

From the bound on the variation the number of jumps that in magnitude exceeds $c=\min _{i=1, \ldots, n-1}\left\{\left|a_{i}-a_{i+1}\right|\right\}$ is bounded by $n_{0}$, where $n_{0}>\left[\operatorname{Var} u^{0}(x)\right] / c$. Hence if the number of shocks becomes unbounded, then for some $a_{j}$ the number of shocks such that the values of $u(t, x)$ to the left and to the right of the shock lie in 
$\left(a_{j-1}, a_{j+1}\right)$ must become arbitrarily large. To see that this cannot happen, divide $(-\infty, \infty)$ into three categories, $\mathrm{A}, \mathrm{B}, \mathrm{C}$, as follows: let $x \in \mathrm{A}$ iff $u^{0}(x)<a_{j-1}$; let $x \in \mathrm{B}$ iff $a_{j-1}<u^{0}(x)<a_{j+1}$; let $x \in \mathrm{C}$ iff $u^{0}(x)>a_{j+1}$. Choose $\zeta_{1}<\zeta_{2}<\cdots<\zeta_{r}$ such that all $x \in\left(\zeta_{i}, \zeta_{i+1}\right)$ are in only one of the categories $\mathrm{A}, \mathrm{B}, \mathrm{C}$.

By a jump in $B$ is meant a shock such that the $u$-values to the left and right both lie in $\left(a_{j-1}, a_{j+1}\right)$. By assumption the number of such jumps is unbounded. It can be shown that for the number of $\mathbf{B}$ shocks to become unbounded shocks seeing $\mathbf{B}$ values (i.e., values of $u(t, x)$ lying in $\left.\left(a_{j-1}, a_{j+1}\right)\right)$ to the left and non-B values to the right must meet shocks seeing non-B values to the left and $B$ values to the right, thus eliminating the non-B values between them. However, since there are finitely many non-B groups, only a finite number of B-shocks can be created in this way, giving a contradiction.

We can now state and prove the main theorem of this paper.

THEOREM 4.9. Let $F$ and $G$ satisfy hypotheses I, II, III, and let $u^{0}(x)$ be bounded and measurable. Then the Cauchy problem (1.1), (1.2) has a global weak solution.

Proof. If $u^{0}(x)$ is a step function, then Theorem 4.1 guarantees the existence of the piecewise smooth weak solution satisfying Condition $\mathrm{E}$ in some strip $0 \leqq t \leqq T$, $T>0$. Since $u(T, x)$ is admissible, Theorem 4.8 gives the solution globally. Since a piecewise smooth, global weak solution is available whenever the initial data is a step function, it is possible to follow the procedure of Douglis [2] and $\mathrm{Wu}$ [5], as outlined in $\S 3$, to obtain the desired result.

\section{BIBLIOGRAPHY}

1. E. Conway and J. Smoller, Global solutions of the Cauchy problem for quasi-linear firstorder equations in several space variables, Comm. Pure Appl. Math. 19 (1966), 95-105. MR 33 \#388.

2. A. Douglis, An ordering principle and generalized solutions of certain quasi-linear partial differential equations, Comm. Pure Appl. Math. 12 (1959), 87-112. MR 21 \#3650.

3. P. D. Lax, Hyperbolic systems of conservation laws. II, Comm. Pure Appl. Math. 10 (1957), 537-566. MR 20 \#176.

4. O. A. Oleĭnik, Discontinuous solutions of non-linear differential equations, Uspehi Mat. Nauk 12 (1957), no. 3 (75), 3-73; English transl., Amer. Math. Soc. Transl. (2) 26 (1963), 95-172. MR 20 \#1055; MR 27 \#1721.

5. Wu Cho-Chün, On the existence and uniqueness of the generalized solutions of the Cauchy problem for quasilinear equations of first order without convexity conditions, Acta. Math. Sinica 13 (1963), 515-530= Chinese Math.-Acta 4 (1964), 561-577.

BROWN UNIVERSITY,

Providence, Rhode IsLAND 02912 